\title{
Monitoring the plate boundary in Tokai area using ACROSS -Role of Toyohashi ACROSS source-
}

\author{
Toshiki WATANABE ${ }^{1}$, Koshun YAMAOKA ${ }^{1}$, Kenji YAMAZAKI $^{2}$ and Yuri SUZUKI ${ }^{1}$ \\ ${ }^{1}$ Graduate School of Environmental Studies, Nagoya University, Japan. \\ ${ }^{2}$ Mitsubishi Corporation Exploration Co., Ltd., Japan,
}

\begin{abstract}
We have been studying long-term continuous monitoring of seismological processes occurring along and around subducting plate boundary using ACROSS (Accurately-Controlled, Routinely-Operated Signal System). In the Tokai region, three ACROSS sources at Toki, Morimachi and Toyohashi are in operation and concentrated efforts are made for the active subsurface monitoring. Using the continuous seismic record observed at Horai seismic array, it is proved that the signal from each ACROSS source is separated without crosstalk, although the sources are operated simultaneously and continuously. The Toyohashi source is located at preferable place to detect and monitor Tokai slow slip event. Using the signals from the Toyohashi source observed by the Hi-net stations, stacking time and $\mathrm{S} / \mathrm{N}$ ratio of selected stations are evaluated.
\end{abstract}

\section{INTRODUCTION}

Time-variant phenomena along the subducting plate boundary with different time constant, such as stress accumulation at asperities, long-term and short-term aseismic slip, deep low-frequency tremors (DLFTs), are ongoing processes and of interest among seismologists. Active seismic monitoring will provide important information on the geomechanical and hydrogeological contact conditions of the plate boundary to understand the mechanism of earthquake generation ${ }^{1)}$

Figure 1 illustrates the concept of active seismic monitoring of the plate coupling and the associated activities along the plate boundary using ACROSS (Accurately Controlled, Routinely Operated Signal System).

ACROSS is based on the precisely repeated transmission of a controlled frequency-modulated (FM) signal and long-term continuous observation synchronized with GPS clock $^{2)}$. The accurate source control enables improving the $\mathrm{S} / \mathrm{N}$ ratio by stacking the observed signals, resulting long distance and deep illumination. The accuracy also enables a "frequency comb" technique, an interesting and effective signal operation in the frequency domain.

\section{ACROSS IN TOKAI AREA}

Figure 2 shows a map of the study area, Tokai region. Three ACROSS sources are currently in operation, named "Toki", "Morimachi" and "Toyohashi",

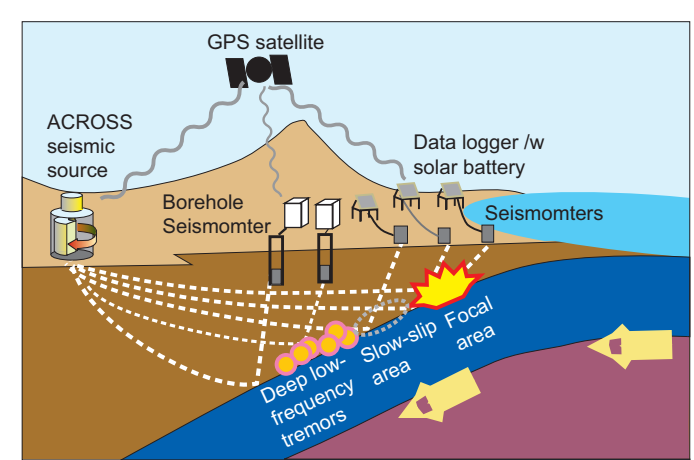

Figure 1. Concept of active seismic monitoring of the plate boundary using seismic ACROSS.

operated by JAEA, JMA and Nagoya University, respectively, to monitor the temporal changes of subsurface properties and conditions. No other area in the world has ongoing concentrated effort on active seismic monitoring.

Figure 3 shows the theoretical spectrum of the force generated by the ACROSS source. The sources uses overlaped frequency-band with each other. Although the sources are separated at $40-80 \mathrm{~km}$, the signals from sources may interfere at the seismic stations.

Since ACROSS is accurately-controlled, a novel technique similar to "frequency comb" as in laser physics can be applied to avoid interference among the signals. The ACROSS sources in Tokai area are operated based on the transmission protocol $^{3)}$ shown in Table 1. 


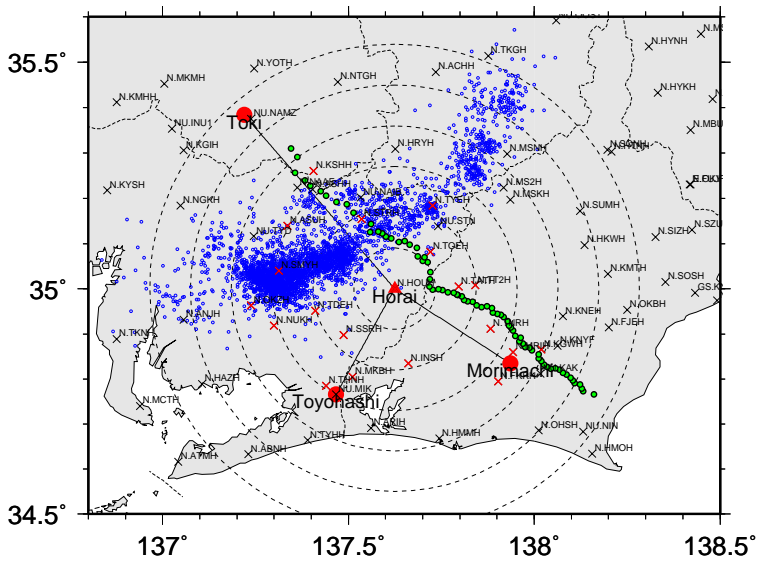

Figure 2. Locations of ACROSS sources at Toki, Morimachi and Toyohashi. Broken circles indicate the distance from Horai with $10 \mathrm{~km}$ interval where the seismic array measurement is ongoing. Open circles indicate the 2008-2009 Kakegawa-Mizunami seismic observation line. Thin small circles show the epicenters of low-frequency earthquakes on the JMA earthquake catalog during 2000 and 2009.
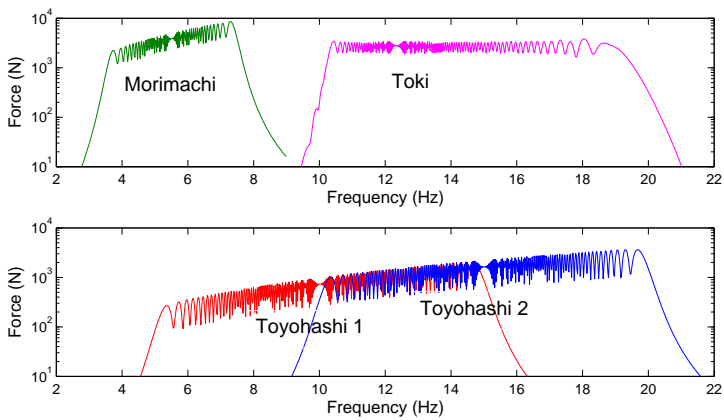

Figure 3. Spectrum of the theoretically designed force of ACROSS sources.

\section{SIGNAL SEPARATION USING HORAI ARRAY OBSERVATION}

Nagoya University has a continuing effort for maintaining a middle-size surface seismic array called "Horai array"4) in Aichi Prefecture shown in Figure 2. Using the continuous seismic data observed by the array, the separation and identification of the signals of each ACROSS source are investigated ${ }^{5)}$.

Figure 4(a) shows the spectrum of the stacked seismic record (3 months) observed at the surface seismometer (HOU08). Figures (b)-(e) shows the extracted line spectra assigned to each ACROSS source. ACROSS signals are visible compared to noises where no ACROSS signal is assigned. Comparison to the spectra of the source signals apparently indicates that the ACROSS signals are separated without crosstalk.

Then, the transfer functions (equivalent to the seismic waveforms) were retrieved from the separated ob-
Table 1. Frequency channel assignment for the ACROSS sources based on Tokai operational protocol $^{3)}$.

\begin{tabular}{|c|c|l|}
\hline Source & Frequency $(\mathrm{Hz})$ & Status \\
\hline MR & $0.0000+0.0200 n$ & Currently not used \\
TK & $0.0025+0.0200 n$ & Temporarily used \\
TK & $0.0050+0.0200 n$ & In operation \\
TY & $0.0075+0.0200 n$ & In operation \\
MR & $0.0100+0.0200 n$ & In operation \\
- & $0.0125+0.0200 n$ & Noise evaluation \\
TY & $0.0150+0.0200 n$ & In operation \\
- & $0.0175+0.0200 n$ & Noise evaluation \\
\hline
\end{tabular}

$n$ : Natural number

MR: Morimachi, TK: Toki, TY: Toyohashi.

(a)

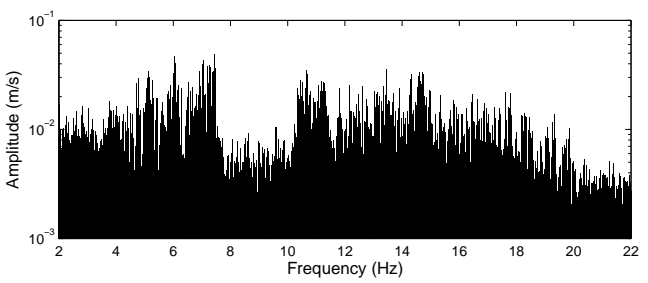

(b)

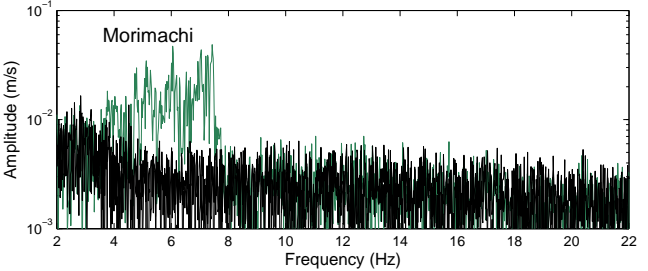

(c)

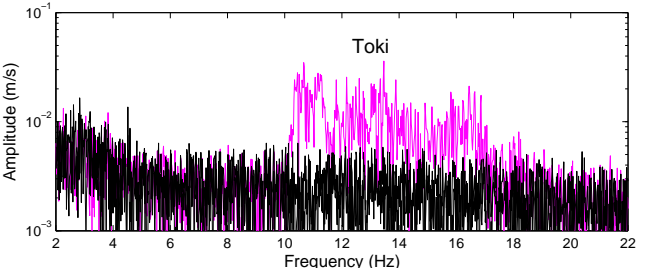

(d)

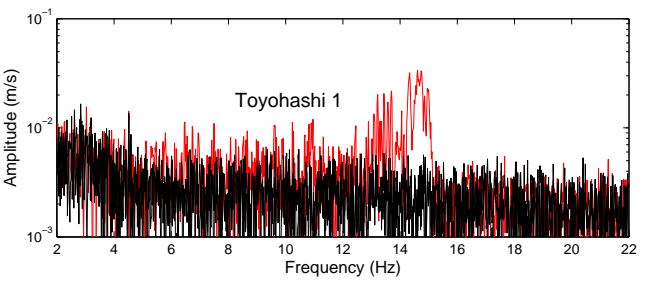

(e)

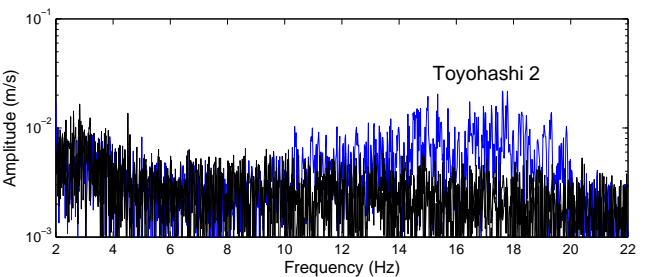

Figure 4. Spectra of the seismic signal obtained by stacking the observed seismic record at Horai for 3 months. (a) Spectrum of the signal, (b)-(e) extracted frequency component assigned to each ACROSS source. Noise components are also shown to evaluate the $\mathrm{S} / \mathrm{N}$ ratio. 

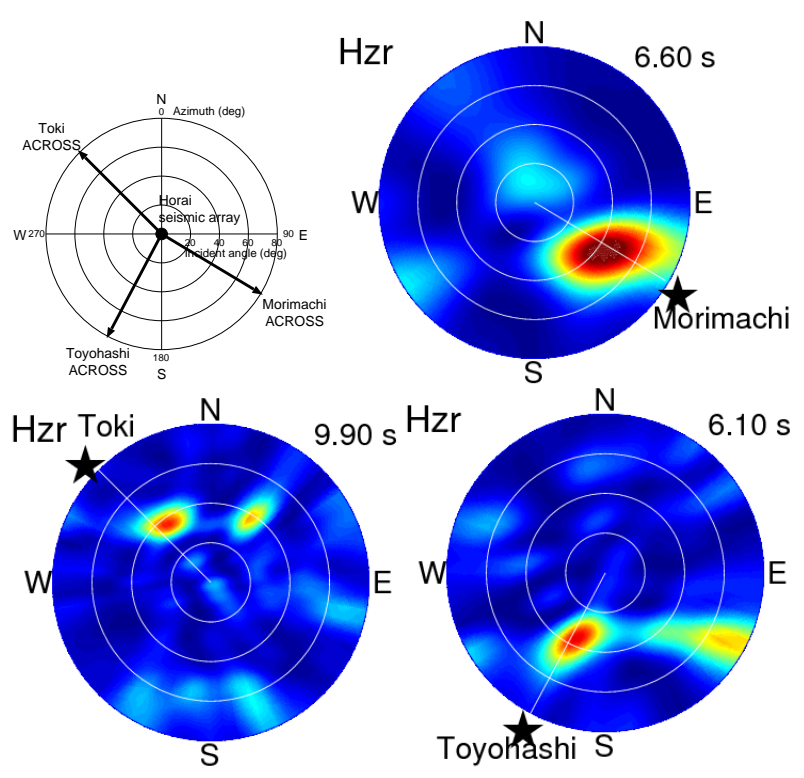

Figure 5. Estimation of the azimuth and incident angle of the P-wave arrival of the transfer function from each ACROSS source by semblance analysis.

served spectra. The semblance analysis are applied to the array of transfer functions in order to search the azimuth and incident angle of each wave packets. Figure 5 indicates that the azimuth of the P-wave arrivals points the direction of the source. This clearly proves that the signals from each source is separated with no crosstalk as designed.

\section{TOYOHASHI ACROSS FOR MONI- TORING SLOW-SLIP}

In 2000, a slow slip event (SSE), a long-term aseismic slip along the plate boundary, occurred beneath Lake Hamana and lasted until 2005. Figure 6 shows the slip distribution of the Tokai slow-slip ${ }^{6}$. The Toyohashi ACROSS source is located in the area that shows the highest slip rate. The location also coincides to the location that shows the largest slip at the earliest stage of the slow-slip event ${ }^{7)}$. Therefore, the Toyohashi ACROSS source is thought to be at the suitable location to detect and monitor the next slow slip event possibly occurs in 5-10 years later under the assumption of event recurrence.

We used the continuous seismic record of Hi-net stations within $50 \mathrm{~km}$ distance from the Toyohashi ACROSS source and examine the detectability of the signal from the source ${ }^{8)}$. The signals from other ACROSS sources were eliminated as discussed in the previous section. Figure 7 shows examples of the result of the signal analysis observed at two Hi-net stations (N.TYHH and N.TOEH). The top figures show that one day stacking is enough for a nearby station (N.TYNH) whereas a distant station (N.TOEH) re-

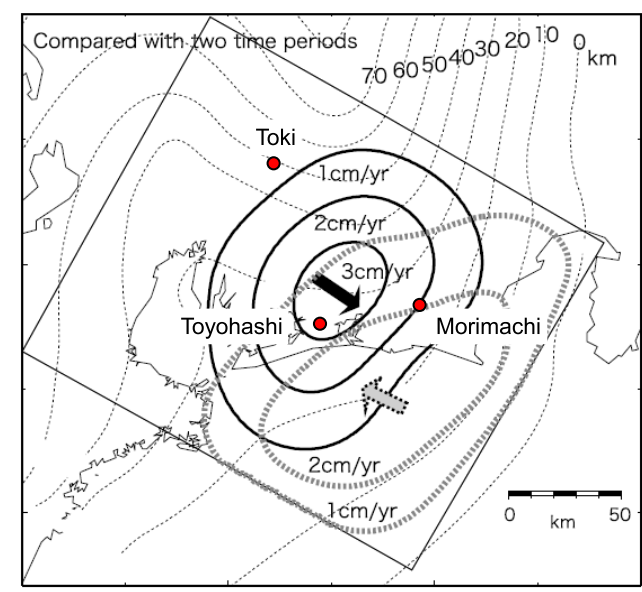

Figure 6. Slip distribution of the latest Tokai slow-slip during January 2001 to December 2002 (Ohta et al., 2002). The location of the ACROSS sources are added to the original figure.

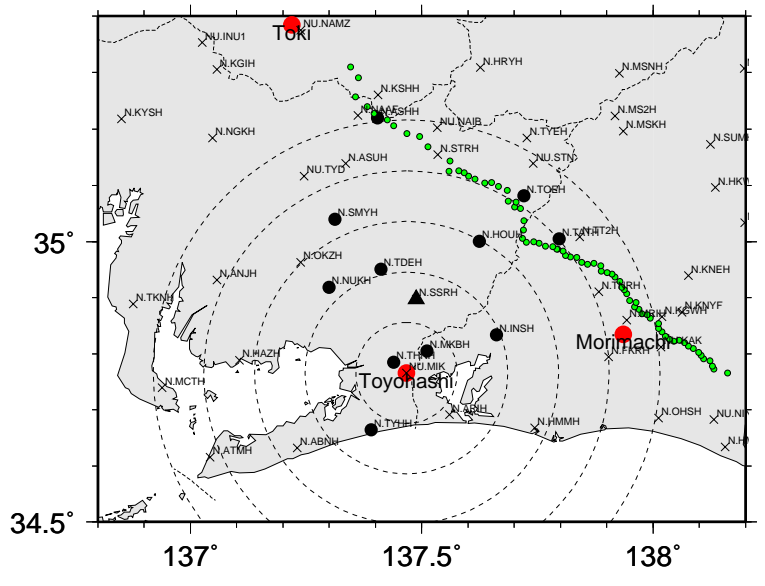

Figure 8. Hi-net stations that are possible to utilize for monitoring slow-slip area (circles) and potential stations (triangles). Broken circles indicate the distance from the Toyohashi ACROSS source with $10 \mathrm{~km}$ interval.

quires 5 months to obtain a $\mathrm{S} / \mathrm{N}$ ratio to detect the signal from the source.

Figure 8 shows our preliminary evaluation which Hi-net station can be used for our purpose. Among those, N.THNH and N.MKBH is the most important for the following two reasons. The one is because these stations lie within a region that showed the highest slip rate at the time of the last event. The other reason is their short stacking time required to obtain necessary $\mathrm{S} / \mathrm{N}$ ratio since they locate close to the Toyohashi ACROSS source. The required stacking time are estimated as one day (N.THNH) and one week (N.MKBH).

\section{CONCLUSIONS}

Using the continuous seismic record observed at Horai seismic array, the signals from three ACROSS 

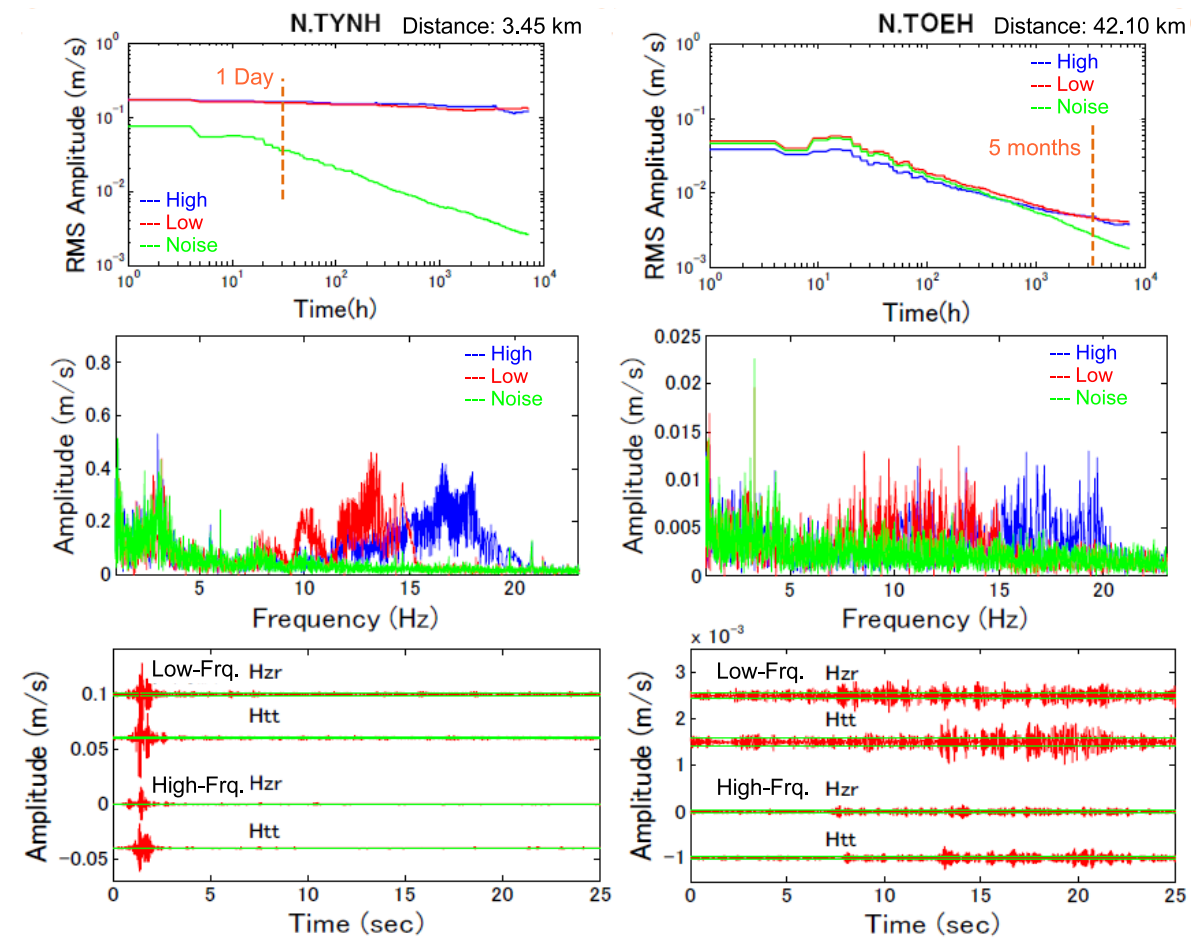

Figure 7. Signal analysis from the Toyohashi ACROSS source observed at Hi-net stations (N.TYHH and N.TOEH). Time evolution of the RMS amplitude of the stacked signal (top), Spectrum (middle) and transfer functions with noise level (bottom).

sources were clearly separated without crosstalk, although the sources are operated simultaneously and continuously. To detect and monitor the next Tokai slow slip event, the Toyohashi source can play important role with the combined use of Hi-net stations, especially N.THNH and N.MKBH.

ACKNOWLEDGMENT We are grateful to T. Kunitomo (JAEA and Shizuoka Univ.), Y. Yoshida and A. Katsumata (JMA) for maintaining the Toki and Morimachi ACROSS sources, respectively and exchanging data and information. We are grateful to M. Kumazawa, N. Fujii, J. Kasahara, R. Ikuta, K. Tsuruga, Y. Hasada and T. Furukawa. We are grateful to Aichi Prefecture Forest Park Office for their cooperation in the field observation and Y. Yamanaka, H. Nakamichi, S. Horikawa and the people in RSVD, Nagoya University for maintaining Horai array and discussion and suggestions.

\section{REFERENCE}

1) Kasahara, J., Tsuruga, K., Hasada, Y., Yamaoka, K., Fujii, N., Yoshida, Y., Kunitomo, T., and Kumazawa, M., A proposal of imaging of the plate boundary using the active monitoring method, Proc. 1st Intern. WS on Active Monitoring in the Solid Earth Geophysics (IWAM04), S2-03, 2004 .

2) Yamaoka, K., Kunitomo, T., Miyakawa, K., Kobayashi K. and Kumazawa, M., A trial for monitoring temporal variation of seismic velocity with ACROSS system, The Island Arc, 10, 336-347, 2001.
3) Kunitomo, T., A draft proposal of transmission protocol on seismic ACROSS in the central Japan, $A b$ stracts, SSJ 2006 Fall meeting, C027, 2006 (in Japanese).

4) Watanabe, T., Ikuta, R., Yamaoka, K., Soma, T. and Fujii, N., Continuous active seismic observation using ACROSS source and seismometer array - Toward active monitoring of Tokai seismogenic area-, Proc. 8th SEGJ Intern. Sympo., 369-372, 2006.

5) Yamazaki, K., Watanabe, T., Yamaoka, Ikuta, R., K., Kunitomo, T., Yoshida, Y., Katsumata, A., Yamanaka, Y., Nakamichi, H. and Horikawa, S., ACROSS signal analysis from Toki, Morimachi and Toyohashi sources using continuous seismic records observed at Horai seismic array, Proc. 122nd SEGJ Conference, 115-118, 2010 (in Japanese).

6) Ohta, Y., Kimata, F. and Sagiya, T, Reexamination of the interplate coupling in the Tokai region, central Japan, based on the GPS data in 1997-2002, Geophys. Res. Lett., 31, L24604, 2004.

7) Ozawa, S., Murakami, M., Kaidzu, M., Tada, T., Sagiya, T., Hatanaka, y., Yarai, H., Nishimura, T., Detection and monitoring of ongoing aseismic slip in the Tokai region, central Japan, Science, 298, 5595, 1009-1012, 2002.

8) Suzuki, Y., Watanabe, T., Yamaoka, K., Kunitomo, T., Yamazaki, K. and Ikuta, R., Estimation of signal detection area from Toyohashi AACROSS by Hinet stations for monitoring of next Tokai slow slip, Abstracts, SSJ 2010 Fall meeting, P3-06, 2010 (in Japanese). 